\title{
Heisenberg uncertainty principles for an oscillatory integral operator
}

L. P. Castro, R. C. Guerra, and N. M. Tuan

Citation: 1798, 020037 (2017); doi: 10.1063/1.4972629

View online: http://dx.doi.org/10.1063/1.4972629

View Table of Contents: http://aip.scitation.org/toc/apc/1798/1

Published by the American Institute of Physics 


\title{
Heisenberg Uncertainty Principles for an Oscillatory Integral Operator
}

\author{
L. P. Castro ${ }^{1, a), b)}$, R. C. Guerra ${ }^{1, c)}$ and N. M. Tuan ${ }^{2, d)}$ \\ ${ }^{1}$ CIDMA - Center for Research and Development in Mathematics and Applications, Department of Mathematics, \\ University of Aveiro, 3810-193 Aveiro, Portugal. \\ ${ }^{2}$ Department of Mathematics, College of Education, Viet Nam National University, G7 Build., 144 Xuan Thuy Rd., \\ Cau Giay Dist., Hanoi, Vietnam.

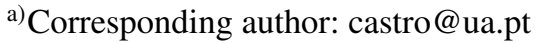 \\ b)URL: http://sweet.ua.pt/castro/ \\ c) ritaguerra@ua.pt \\ d)nguyentuan@vnu.edu.vn
}

\begin{abstract}
The main aim of this work is to obtain Heisenberg uncertainty principles for a specific oscillatory integral operator which representatively exhibits different parameters on their sine and cosine phase components. Additionally, invertibility theorems, Parseval type identities and Plancherel type theorems are also obtained.
\end{abstract}

\section{INTRODUCTION}

Uncertainty principles of Heisenberg type are well-known to have strong consequences in different subareas of Physics and Mathematics. More specifically, within quantum mechanics, the uncertainty principle is basically the translation of a characteristic feature of quantum mechanical systems; from the mathematical point of view, this may be realized by a specific inequality which, e.g., for the Fourier transform, basically states that a nonzero function and its Fourier transform cannot both be sharply localized. This fact is of significative importance e.g. in the theory of partial differential equations (cf. [2]).

Heisenberg's original proposal was given in very generic terms. It was basically concentrated on qualitative examples which would allow an understanding of simple experiments in quantum mechanics. The first mathematically exact formulation of the Heisenberg uncertainty relations is due to Kennard [7]. The inequality of Kennard was generalized in 1929 by Robertson [8] (by englobing self-adjoint operators), and this was also generalized, later on, in 1930, by Schrödinger [10]. Anyway, many variations on Heisenberg's inequality have been developed during the last century. We refer to $[2,3,4,5,6,7,11,12,13]$ for the seminal paper of Heisenberg, as well as for some of the subsequent related fundamental works and surveys. In particular, Heisenberg uncertainty principles for integral operators are known to be important e.g. in the interpretation of the behaviour of this type of operators and have consequent influences in their applications.

The main object of this work is the oscillatory integral operator $T$, defined in the following way in the framework of the $L^{2}\left(\mathbb{R}^{d}\right)$ space,

$$
(T f)(x)=\frac{1}{(2 \pi)^{\frac{d}{2}}} \int_{\mathbb{R}^{d}}[2 \cos (x y)+i \sin (x y)] f(y) d y,
$$

where the different coefficients on the cosine and sine functions are chosen as a representative case of an unbalanced weight on the corresponding oscillation. In this work we will derive such type of uncertainty principles for the operator (1). Anyway, prior to that, we will analyse more basic properties of (1). Namely, we will prove its inversion formula, and obtain a Plancherel theorem and Parseval type identities. Moreover, we will add an example of a classical partial differential equation which can be treated in an efficient way by using our operator. In the next section, we can see 
that $T$ is an invertible, non-unitary and asymmetric, continuous linear operator in $L^{2}\left(\mathbb{R}^{d}\right)$, and it is also well-defined on $L^{1}\left(\mathbb{R}^{d}\right)$ with an explicit inversion formula.

\section{FUNDAMENTAL PROPERTIES}

We start by indicating some of the notation that we will be using. Let $(F f)(x)=\frac{1}{(2 \pi)^{d / 2}} \int_{\mathbb{R}^{d}} e^{-i x y} f(y) d y$ and $\left(F^{-1} f\right)(x)=$ $\frac{1}{(2 \pi)^{d / 2}} \int_{\mathbb{R}^{d}} e^{i x y} f(y) d y$ denote the Fourier and inverse Fourier transform, respectively. Throughout the paper we write $\mathbb{N}_{0}:=\{0,1,2, \ldots\}$. If $\alpha=\left(\alpha_{1}, \ldots, \alpha_{d}\right)$ is a $d$-tuple of non-negative integers $\alpha_{k}, k=1, \ldots, d$, we call $\alpha$ a multi-index, and $|\alpha|:=\alpha_{1}+\cdots+\alpha_{d}$. For $\beta=\left(\beta_{1}, \ldots, \beta_{d}\right)$, we consider $D_{x}^{\beta}=\frac{\partial^{\beta}}{\partial x_{1}^{\beta_{1}} \ldots \partial x_{d}^{\beta_{d}}}$. The Schwartz space will be denoted by $\mathcal{S}$.

The multi-dimensional Hermite functions are defined by $\Phi_{\alpha}(x):=(-1)^{|\alpha|} e^{\frac{1}{2}|x|^{2}} D_{x}^{\alpha} e^{-|x|^{2}}$ (see [15]). The Hermite functions are essential in several applications, as it is the case of the study of the celebrated harmonic oscillator in quantum mechanics. To begin with, we formulate a theorem relating those functions to our operator $T$.

Theorem 1 The following formula holds

$$
T \Phi_{\alpha}=\left\{\begin{array}{cccc}
(-1)^{\frac{|\alpha|}{2}} 2 \Phi_{\alpha}, & \text { if } & |\alpha| \equiv 0,2 & (\bmod 4) \\
(-1)^{\frac{|\alpha|-1}{2}} i \Phi_{\alpha}, & \text { if } & |\alpha| \equiv 1,3 & (\bmod 4)
\end{array}\right.
$$

Proof. If we consider $L^{1}\left(\mathbb{R}^{d}\right)$ as the domain of the Fourier and inverse Fourier transform $F$ and $F^{-1}$, respectively, then the domain of $T$ is also $L^{1}\left(\mathbb{R}^{d}\right)$. In fact, we can rewrite $T$ as $T=\frac{1}{2} F+\frac{3}{2} F^{-1}$. Since $F \Phi_{\alpha}=(-i)^{|\alpha|} \Phi_{\alpha}$ and $F^{-1} \Phi_{\alpha}=i^{|\alpha|} \Phi_{\alpha}$ (see [16]), we have $T \Phi_{\alpha}=\left[\frac{1}{2}(-i)^{|\alpha|}+\frac{3}{2} i^{|\alpha|}\right] \Phi_{\alpha}$. Calculating the coefficient on the right-hand side of this equality we obtain (2). The theorem is proved.

The following auxiliary lemma is very useful for proving some of our further theorems.

Lemma 2 (cf. [16]) The formula $\frac{1}{2}\{f(x+0)+f(x-0)\}=\lim _{\lambda \rightarrow \infty} \frac{1}{\pi} \int_{-\infty}^{+\infty} f(t) \frac{\sin (\lambda(x-t))}{x-t} d t$ holds if $\frac{f(x)}{1+|x|} \in L^{1}(\mathbb{R})$.

The operator $T$ also has its Riemann-Lebesgue lemma which can be proved in a straightforward way.

Theorem 3 (Riemann-Lebesgue Lemma) $\quad T$ is a bounded linear operator from $L^{1}\left(\mathbb{R}^{d}\right)$ into $C_{0}\left(\mathbb{R}^{d}\right)$. Namely, if $f \in L^{1}\left(\mathbb{R}^{d}\right)$, then $T f \in C_{0}\left(\mathbb{R}^{d}\right)$ and $\|T f\|_{\infty} \leq \frac{\sqrt{5}}{(2 \pi)^{\frac{d}{2}}}\|f\|_{1}$, where $\|\cdot\|_{\infty}$ and $\|\cdot\|_{1}$ are the usual supremum and $L^{1}-$ norms, respectively.

Proof. By using the Riemann-Lebesgue lemma for the integral transforms with each one of cosine and sine kernels, we see that $T f \in C_{0}\left(\mathbb{R}^{d}\right)$, provided $f \in L^{1}\left(\mathbb{R}^{d}\right)$. Moreover, by the Cauchy-Schwartz inequality we have

$$
\begin{aligned}
\|T f\|_{\infty} & =\sup _{x \in \mathbb{R}^{d}}\left|\frac{1}{(2 \pi)^{\frac{d}{2}}} \int_{\mathbb{R}^{d}}[2 \cos (x y)+i \sin (x y)] f(y) d y\right|=\sup _{x \in \mathbb{R}^{d}} \frac{1}{(2 \pi)^{\frac{d}{2}}} \int_{\mathbb{R}^{d}}|2 \cos (x y)+i \sin (x y)||f(y)| d y \\
& \leq \sup _{x \in \mathbb{R}^{d}} \frac{1}{(2 \pi)^{\frac{d}{2}}} \int_{\mathbb{R}^{d}} \sqrt{[4+1]\left[\cos ^{2}(x y)+\sin ^{2}(x y)\right]}|f(y)| d y=\frac{\sqrt{5}}{(2 \pi)^{\frac{d}{2}}}\|f\|_{1} .
\end{aligned}
$$

Theorem $4 T$ is a continuous linear operator of $\mathcal{S}$ into itself, and fulfills the polynomial identity: $T^{4}-3 T^{2}-4 I=0$, where I is the identity operator. Moreover, the operator $T$ is invertible in $\mathcal{S}$.

Proof. Clearly, $T$ is a continuous operator in $\mathcal{S}$. We shall prove the polynomial identity. Let us first prove the identity $\left(T^{2} f\right)(x)=\frac{3}{2} f(x)+\frac{5}{2} f(-x)$, for every $f \in \mathcal{S}$. For $\lambda>0$, consider the $d$-dimensional box in $\mathbb{R}^{d}: B(0, \lambda):=$ $\left\{y=\left(y_{1}, \ldots, y_{d}\right) \in \mathbb{R}^{d}:\left|y_{k}\right| \leq \lambda, k=1, \ldots, d\right\}$. Obviously, $\int_{B(0, \lambda)} \cos (x y) \sin (x v) d x=0$. Acting inductively on $d$, we obtain

$$
\int_{B(0, \lambda)} \cos (y(x-t)) d y=\frac{2^{d} \sin \left(\lambda\left(x_{1}-t_{1}\right)\right) \cdots \sin \left(\lambda\left(x_{d}-t_{d}\right)\right)}{\left(x_{1}-t_{1}\right) \cdots\left(x_{d}-t_{d}\right)} .
$$


Since $f \in \mathcal{S}$, we may use the Fubini's theorem, Lemma 2, and (3) to calculate $T^{2} f$, as follows

$$
\begin{aligned}
\left(T^{2} f\right)(x) & =\lim _{\lambda \rightarrow \infty} \frac{1}{(2 \pi)^{d}} \int_{\mathbb{R}^{d}}[2 \cos (x y)+i \sin (x y)] d y \int_{B(0, \lambda)}[2 \cos (y t)+i \sin (y t)] f(t) d t \\
& =\lim _{\lambda \rightarrow \infty} \frac{1}{(2 \pi)^{d}} \int_{\mathbb{R}^{d}} f(t) \int_{B(0, \lambda)}\left[\frac{5}{2} \cos (y(x+t))+\frac{3}{2} \cos (y(x-t))+2 i \sin (y(x+t))\right] d y d t \\
& =\lim _{\lambda \rightarrow \infty} \frac{1}{(2 \pi)^{d}} \int_{\mathbb{R}^{d}} f(t) \int_{B(0, \lambda)}\left[\frac{5}{2} \cos (y(x+t))+\frac{3}{2} \cos (y(x-t))\right] d y d t \\
& =\lim _{\lambda \rightarrow \infty} \frac{1}{(2 \pi)^{\frac{d}{2}}} \int_{\mathbb{R}^{d}}\left[\frac{3}{2} \frac{2^{d} \sin \left(\lambda\left(y_{1}-t_{1}\right)\right) \cdots \sin \lambda\left(y_{d}-t_{d}\right)}{\left(y_{1}-t_{1}\right) \cdots\left(y_{d}-t_{d}\right)}+\frac{5}{2} \frac{2^{d} \sin \left(\lambda\left(y_{1}+t_{1}\right)\right) \cdots \sin \left(\lambda\left(y_{d}+t_{d}\right)\right)}{\left(y_{1}+t_{1}\right) \cdots\left(y_{d}+t_{d}\right)}\right] f(t) d t \\
& =\frac{3}{2} f(x)+\frac{5}{2} f(-x), \quad x \in \mathbb{R}^{d} .
\end{aligned}
$$

Thus, we have

$$
\begin{aligned}
\left(T^{4} f\right)(x) & =T^{2}\left[\left(T^{2} f\right)(x)\right]=T^{2}\left[\frac{3}{2} f(x)+\frac{5}{2} f(-x)\right]=\frac{3}{2}\left[\frac{3}{2} f(x)+\frac{5}{2} f(-x)\right]+\frac{5}{2}\left[\frac{3}{2} f(-x)+\frac{5}{2} f(x)\right] \\
& =\frac{34}{4} f(x)+\frac{30}{4} f(-x), \quad x \in \mathbb{R}^{d} .
\end{aligned}
$$

Combining (4) and (5), we get $T^{4} f-3 T^{2} f-4 f=0$, for every $f \in \mathcal{S}$. The polynomial identity for $T$ is proved.

By this polynomial identity, we obtain $T\left[\frac{1}{4} T^{3}-\frac{3}{4} T\right]=\left[\frac{1}{4} T^{3}-\frac{3}{4} T\right] T=I$, which implies that $T$ is invertible by $T^{-1}=\frac{1}{4} T^{3}-\frac{3}{4} T$. The proof of Theorem 4 is complete.

The invertibility of $T$ in $\mathcal{S}$ is a key ingredient to prove the inversion theorem below, simply because $\mathcal{S}$ is dense in $L^{1}\left(\mathbb{R}^{d}\right)$. Moreover, we will provide the explicit inversion formula in the next identity (6).

Theorem 5 (Inversion Theorem) If $f \in L^{1}\left(\mathbb{R}^{d}\right)$, and if $T f \in L^{1}\left(\mathbb{R}^{d}\right)$, then

$$
f_{0}(x):=\frac{1}{(2 \pi)^{\frac{d}{2}}} \int_{\mathbb{R}^{d}}(T f)(y)\left[\frac{1}{2} \cos (x y)-i \sin (x y)\right] d y=f(x), \quad \text { for almost every } x \in \mathbb{R}^{d} .
$$

Proof. Let us first prove the inversion formula in $\mathcal{S}$, i.e.

$$
g(x)=\frac{1}{(2 \pi)^{\frac{d}{2}}} \int_{\mathbb{R}^{d}}(T g)(y)\left[\frac{1}{2} \cos (x y)-i \sin (x y)\right] d y, \quad \text { for every } x \in \mathbb{R}^{d}, \quad g \in \mathcal{S} .
$$

Indeed, since $\operatorname{Tg} \in \mathcal{S}$, the inner function on the right-hand side of (7) belongs to $\mathcal{S}$. This means that the integral (7) is uniformly convergent on $\mathbb{R}^{d}$ according to each variable $x_{1}, \ldots, x_{d}$. Let us calculate the right-hand side of (7), using Fubini's theorem, Lemma 2 and (3). So, we have

$$
\begin{aligned}
\frac{1}{(2 \pi)^{\frac{d}{2}}} \int_{\mathbb{R}^{d}}(T g)(y) & {\left[\frac{1}{2} \cos (x y)-i \sin (x y)\right] d y=\lim _{\lambda \rightarrow \infty} \frac{1}{(2 \pi)^{\frac{d}{2}}} \int_{B(0, \lambda)}(T g)(y)\left[\frac{1}{2} \cos (x y)-i \sin (x y)\right] d y } \\
& =\lim _{\lambda \rightarrow \infty} \frac{1}{(2 \pi)^{d}} \int_{\mathbb{R}^{d}}\left[\frac{1}{2} \cos (x y)-i \sin (x y)\right] \int_{B(0, \lambda)}[2 \cos (y t)+i \sin (y t)] g(t) d t d y \\
& =\lim _{\lambda \rightarrow \infty} \frac{1}{(2 \pi)^{d}} \int_{\mathbb{R}^{d}} g(t) \int_{B(0, \lambda)}\left[\frac{1}{2} \cos (x y)-i \sin (x y)\right][2 \cos (y t)+i \sin (y t)] d y d t \\
& =\lim _{\lambda \rightarrow \infty} \frac{1}{(2 \pi)^{d}} \int_{\mathbb{R}^{d}} g(t)\left(\int_{B(0, \lambda)}(\cos y(x-t)) d y d t\right) \\
& =\frac{1}{(2 \pi)^{d}} \lim _{\lambda \rightarrow \infty} \int_{\mathbb{R}^{d}} g(t) \frac{2^{d} \sin \left(\lambda\left(x_{1}-t_{1}\right)\right) \cdots \sin \left(\lambda\left(x_{d}-t_{d}\right)\right)}{\left(x_{1}-t_{1}\right) \cdots\left(x_{d}-t_{d}\right)} d t=g(x),
\end{aligned}
$$


for every $x \in \mathbb{R}^{d}$ (having in mind that $g \in \mathcal{S}$ ). Identity (7) is proved. Now let $f \in L^{1}\left(\mathbb{R}^{d}\right)$, and let $g \in \mathcal{S}$. Clearly, $\int_{\mathbb{R}^{d}} f(x)(T g)(x) d x=\int_{\mathbb{R}^{d}} g(y)(T f)(y) d y$. Hence,

$$
\begin{aligned}
\int_{\mathbb{R}^{d}} f(x)(T g)(x) d x & =\frac{1}{(2 \pi)^{\frac{d}{2}}} \int_{\mathbb{R}^{d}}\left(\int_{\mathbb{R}^{d}}(T g)(x)\left[\frac{1}{2} \cos (x y)-i \sin (x y)\right] d x\right)(T f)(y) d y \\
& =\int_{\mathbb{R}^{d}}(T g)(x)\left(\frac{1}{(2 \pi)^{\frac{d}{2}}} \int_{\mathbb{R}^{d}}(T f)(y)\left[\frac{1}{2} \cos (x y)-i \sin (x y)\right] d y\right) d x=\int_{\mathbb{R}^{d}} f_{0}(x)(T g)(x) d x .
\end{aligned}
$$

By (7) the functions $T g$ cover all $\mathcal{S}$. Therefore, $\int_{\mathbb{R}^{d}}\left(f_{0}(x)-f(x)\right) \Psi(x) d x=0$, for every $\Psi \in \mathcal{S}$. Since $\mathcal{S}$ is dense in $L^{1}\left(\mathbb{R}^{d}\right)$, it follows $f_{0}(x)-f(x)=0$ for almost every $x \in \mathbb{R}^{d}$. Theorem 5 is proved.

Corollary 6 (Uniqueness Theorem) If $f \in L^{1}\left(\mathbb{R}^{d}\right)$, and if $T f=0$, then $f=0$.

Theorem 4 leads us to a Plancherel theorem for $T$ (as $\mathcal{S}$ is dense in $L^{2}\left(\mathbb{R}^{d}\right)$ ).

Theorem 7 (Plancherel Theorem) There is a linear isomorphic operator $\bar{T}$ from $L^{2}\left(\mathbb{R}^{d}\right)$ onto itself which is uniquely determined by $\bar{T} f=T f$, for every $f \in \mathcal{S}$.

The extension operator satisfies the polynomial identity $\bar{T}^{4}-3 \bar{T}^{2}-4 I=0$.

Proof. Recall that $S$ is dense in $L^{2}\left(\mathbb{R}^{d}\right)$. As the map $f \mapsto T f$ is continuous (relatively to the $L^{2}$-metric) of the dense subspace $\mathcal{S}$ of $L^{2}\left(\mathbb{R}^{d}\right)$ onto $\mathcal{S}$, it has a unique continuous extension $\bar{T}: L^{2}\left(\mathbb{R}^{d}\right) \rightarrow L^{2}\left(\mathbb{R}^{d}\right)$ (see [9]). Therefore, the polynomial identity $\bar{T}^{4} f-3 \bar{T}^{2} f-4 f=0\left(\right.$ in $L^{2}\left(\mathbb{R}^{d}\right)$ ) follows directly from Theorem 4 , the continuity of $\bar{T}$, and the circumstance that $\mathcal{S}$ is dense in $L^{2}\left(\mathbb{R}^{d}\right)$. Theorem 7 is proved.

Since the uniqueness of the extension and identity (7) holds on the space $\mathcal{S}$, which is dense in $L^{2}\left(\mathbb{R}^{d}\right)$, we can state the Plancherel theorem in a clearer way. We shall denote by $\langle\cdot, \cdot\rangle_{2}$ the usual inner product of $L^{2}\left(\mathbb{R}^{d}\right)$, and by $\|\cdot\|_{2}$ the corresponding norm.

Theorem 8 (Plancherel Theorem) $\quad T$ defines a bounded linear operator in $L^{2}\left(\mathbb{R}^{d}\right)$, admitting an inverse given by

$$
f(y)=\lim _{k \rightarrow \infty} \frac{1}{(2 \pi)^{\frac{d}{2}}} \int_{|y| \leq k}\left[\frac{1}{2} \cos (x y)-i \sin (x y)\right](T f)(x) d x,
$$

in the sense of strong convergence, this is,

$$
\lim _{k \rightarrow \infty}\left\|\frac{1}{(2 \pi)^{\frac{d}{2}}} \int_{|y| \leq k}\left[\frac{1}{2} \cos (x y)-i \sin (x y)\right](T f)(x) d x-f(y)\right\|_{2}=0 .
$$

In the sequel, we will also denote by $T$ the integral operator defined in the Hilbert space $L^{2}\left(\mathbb{R}^{d}\right)$ - as there is no danger of confusion.

Corollary 9 (Spectrum) $\quad$ (P1) For any $\lambda \notin\{-i, i,-2,2\}$ we have

$$
(T-\lambda I)^{-1}=-\frac{1}{\lambda^{4}-3 \lambda^{2}-4}\left[T^{3}+\lambda T^{2}+\left(\lambda^{2}-3\right) T+\left(\lambda^{3}-3 \lambda\right) I\right] .
$$

(P2) The spectrum of the operator $T$ is given by $\sigma(T)=\{-i, i,-2,2\}$.

Proof. Proposition (P1) is an immediate consequence of the polynomial identity of Theorem 7, and (P2) follows at once from Theorem 1 and from the fact that $\pm i, \pm 2$ are eigenvalues of $T$.

The reflection operator will be denoted by $W$, this is, $(W \varphi)(x):=\varphi(-x)$, for $\varphi \in L^{2}\left(\mathbb{R}^{d}\right)$.

Theorem 10 (Parseval type Identities) The following identities hold for any $f, g \in L^{2}\left(\mathbb{R}^{d}\right)$ :

$$
\langle T f, T g\rangle_{2}=\frac{3}{2}\langle f, g\rangle_{2}+\frac{5}{2}\langle f, W g\rangle_{2} ; \quad\left\langle T^{-1} f, T^{-1} g\right\rangle_{2}=-\frac{3}{8}\langle f, g\rangle_{2}+\frac{5}{8}\langle f, W g\rangle_{2} ; \quad\left\langle T f, T^{-1} g\right\rangle_{2}=\langle f, g\rangle_{2} .
$$


Proof. Let us write $T=\frac{1}{2} F+\frac{3}{2} F^{-1}$. The well-known identities $\langle F f, g\rangle_{2}=\langle f, F g\rangle_{2}$ and $\left\langle F^{-1} f, g\right\rangle_{2}=\left\langle f, F^{-1} g\right\rangle_{2}$, and a straightforward computation yield the just presented identities (9).

Theorem $11 T$ is not unitary in $L^{2}\left(\mathbb{R}^{d}\right)$, being its norm $\|T\|_{2}=2$.

Proof. If $f \in L^{2}\left(\mathbb{R}^{d}\right)$, then $F f \in L^{2}\left(\mathbb{R}^{d}\right)$ and $F^{-1} f \in L^{2}\left(\mathbb{R}^{d}\right)$. In this way, using (9), we obtain

$$
\|T f\|_{2}^{2}=\frac{3}{2}\langle f, f\rangle_{2}+\frac{5}{2}\langle f, W f\rangle_{2}=\frac{3}{2}\|f\|_{2}^{2}+\frac{5}{2}\langle f, W f\rangle_{2} \leq \frac{3}{2}\|f\|_{2}^{2}+\frac{5}{2}\left(\|f\|_{2}\|W f\|_{2}\right)=\frac{3}{2}\|f\|_{2}^{2}+\frac{5}{2}\|f\|_{2}^{2}=4\|f\|_{2}^{2} .
$$

On the other hand, by Theorem 1, we have $T \Phi_{\alpha}=2 \Phi_{\alpha}$ for the Hermite functions $\Phi_{\alpha}$ with $|\alpha|$-even. So, we deduce that $\|T\|_{2}=2$.

We end up this section by exemplifying that similarly to the use of the Fourier transform, it is possible to use $T$ (or $T^{-1}$ ) for solving many linear partial differential equations. We will consider just one typical case of classical partial differential equation. If $x \in \mathbb{R}^{d}$ and if $\alpha \in \mathbb{N}_{0}^{d}$, the monomial $x^{\alpha}$ is defined by $x^{\alpha}=x_{1}^{\alpha_{1}} \cdots x_{d}^{\alpha_{d}}$. For $f \in \mathcal{S}$, we define $g_{\beta}(x)=x^{\beta} f(x)$, where $x \in \mathbb{R}^{d}, \beta \in \mathbb{N}_{0}^{d}$. The function $D_{x}^{\beta}(T f)$ belongs to $\mathcal{S}$, for all $\beta \in \mathbb{N}_{0}^{d}$. The following properties of $T$ are very convenient for using in the partial differential calculus.

Theorem 12 Let $f \in \mathcal{S}$. For $\beta \in \mathbb{N}_{0}^{d}$, the following identity holds

$$
D_{x}^{\beta}(T f)=\left\{\begin{aligned}
(-1) \frac{|\beta|}{2} T g_{\beta}, & \text { if }|\beta| \text { is even } \\
(-1)^{\frac{|\beta|-1}{2}} i S g_{\beta}, & \text { if }|\beta| \text { is odd. }
\end{aligned}\right.
$$

where $S$ is the transform given by

$$
(S f)(x)=\frac{1}{(2 \pi)^{\frac{d}{2}}} \int_{\mathbb{R}^{d}}[\cos (x y)+2 i \sin (x y)] f(y) d y,
$$

which admits the inverse

$$
\left(S^{-1} f\right)(x)=\frac{1}{(2 \pi)^{\frac{d}{2}}} \int_{\mathbb{R}^{d}}\left[\cos (x y)-\frac{i}{2} \sin (x y)\right] f(y) d y .
$$

The proof of (10) can be done through a straightforward computation, and that of the inversion formula (11) can be completed in the same way as in the proof of Theorem 5 . Therefore, we choose not to add the proof of Theorem 12 in here.

Example 13 (see [1, Example 2.12.3], [14, Chapter 5]) Consider the following equation

$$
\frac{\partial u}{\partial t}=\frac{\partial^{2} u}{\partial x^{2}} \quad \text { such that } \quad u(x, 0)=f(x), \quad-\infty<x<\infty, \quad t>0 .
$$

Let $U(\xi, t)=\frac{1}{\sqrt{2 \pi}} \int_{\mathbb{R}} u(x, t)[2 \cos (x \xi)+i \sin (x \xi)] d x$. Integrating twice by parts and assuming that the terms at $+\infty$ and $-\infty$ vanish, we get

$$
\begin{aligned}
\frac{\partial U}{\partial t} & =\frac{1}{\sqrt{2 \pi}} \int_{\mathbb{R}} \frac{\partial u}{\partial t}[2 \cos (x \xi)+i \sin (x \xi)] d x=\frac{1}{\sqrt{2 \pi}} \int_{\mathbb{R}} \frac{\partial^{2} u}{\partial x^{2}}[2 \cos (x \xi)+i \sin (x \xi)] d x \\
& =-\frac{\xi^{2}}{\sqrt{2 \pi}} \int_{\mathbb{R}} u[2 \cos (x \xi)+i \sin (x \xi)] d x=-\xi^{2} U .
\end{aligned}
$$

It implies that $U(\xi, t)=A(\xi) e^{-\xi^{2} t}$. Putting $t=0$, we obtain $A(\xi)=\frac{1}{\sqrt{2 \pi}} \int_{\mathbb{R}} f(x)[2 \cos (x \xi)+i \sin (x \xi)] d x=(T f)(\xi)$. Hence, $U(\xi, t)=(T f)(\xi) e^{-\xi^{2} t}$. Thus, the solution is

$$
u(x, t)=\frac{1}{\sqrt{2 \pi}} \int_{\mathbb{R}}(T f)(\xi) e^{-\xi^{2} t}\left[\frac{1}{2} \cos (x \xi)-i \sin (x \xi)\right] d \xi .
$$

As the functions $2 e^{-\xi^{2} t} \sin (\xi x) \cos (\xi u)$ and $\frac{1}{2} e^{-\xi^{2} t} \sin (\xi u) \cos (\xi x)$ are odd corresponding to the variable $\xi$, the solution can be represented as $u(x, t)=\frac{1}{2 \pi} \int_{\mathbb{R}} \int_{\mathbb{R}} \cos (\xi(x-u)) e^{-\xi^{2} t} f(u) d u d \xi$. 


\section{UNCERTAINTY PRINCIPLES}

In view to derive Heisenberg uncertainty type principles for $T$, we will use the Hermite functions, as it is expected from the knowledge of similar cases; cf. [17]. We will start with the one dimensional case, for the real line, and later on we will consider the $\mathbb{R}^{d}$ case (with $d \geq 1$ ). For the real line case (see e.g. [15] and [16]), the Hermite polynomial of degree $n$ can be defined by

$$
H_{n}(t)=(-1)^{n} e^{t^{2}}\left(\frac{d}{d t}\right)^{n} e^{-t^{2}}, \quad n \in \mathbb{N}_{0},
$$

and we write $\phi_{n}(t):=e^{-\frac{1}{2} t^{2}} H_{n}(t)=(-1)^{n} e^{\frac{1}{2} t^{2}}\left(\frac{d}{d t}\right)^{n} e^{-t^{2}}$, that is a solution of the following differential equation

$$
\frac{d^{2} y}{d t^{2}}-t^{2} y=-(2 n+1) y
$$

For the corresponding orthogonal system on $L^{2}(\mathbb{R})$, we take $\psi_{n}(t):=\phi_{n}(t) /\left(2^{n} n ! \sqrt{\pi}\right)^{\frac{1}{2}}$. We will be able to analyse and use the images of $\psi_{n}$ by our operator $T$. From the fact that $y=\phi_{n}$ is a solution of (13), we obtain the following recurrence relation for the Hermite functions:

$$
\sqrt{2} t \psi_{n}(t)=\sqrt{n+1} \psi_{n+1}(t)+\sqrt{n} \psi_{n-1}(t), \quad n \in \mathbb{N} .
$$

We will admit that $\psi_{-1}=0$. Moreover, by the properties of the Hermite functions, we also know that $\left\langle\psi_{m}, \psi_{n}\right\rangle_{2}=\delta_{m n}$, where $\delta_{m n}$ is the Kronecker symbol.

Our first uncertainty principle of Heisenberg type is now stated in the following theorem.

Theorem 14 If $f \in L^{2}(\mathbb{R})$ and $g=T f$, then

$$
\|t f(t)\|_{2} \cdot\|\xi g(\xi)\|_{2} \geq\|f\|_{2}^{2} .
$$

In view of the next main goal of proving this theorem, let us take a function $f \in L^{2}(\mathbb{R})$ and consider $g=T f$. As $T=\frac{1}{2} F+\frac{3}{2} F^{-1}$, and using (9), we obtain:

$$
\gamma_{n}:=\left\langle f, \psi_{n}\right\rangle_{2}=\left\langle T f, T^{-1} \psi_{n}\right\rangle_{2}=\left\langle g, \frac{3}{4} F \psi_{n}-\frac{1}{4} F^{-1} \psi_{n}\right\rangle_{2}=\frac{3 i^{-n}-i^{n}}{4}\left\langle g, \psi_{n}\right\rangle_{2} .
$$

Equivalently, we have $\left\langle g, \psi_{n}\right\rangle_{2}=\theta_{n} \gamma_{n}$, where $\theta_{n}:=\frac{4}{3 i^{-n}-i^{n}}$, for $n=0,1, \ldots$ In a straightforward way, we obtain

$$
\theta_{n}=\left\{\begin{array}{ccc}
(-1)^{\frac{n}{2}} 2, & n \equiv 0,2 \quad(\bmod 4) \\
(-1)^{\frac{n-1}{2}} i, & n \equiv 1,3 \quad(\bmod 4)
\end{array} \quad, \quad \theta_{n}^{2}=-\theta_{n} \theta_{n+2}=\left\{\begin{array}{rll}
4, & n \equiv 0,2 \quad(\bmod 4) \\
-1, & n \equiv 1,3 \quad(\bmod 4)
\end{array}\right.\right.
$$

Using these facts, we will first prove the following lemma.

Lemma 15 If $f \in L^{2}(\mathbb{R})$ and $g=T f$, then

$$
\|t f(t)\|_{2}^{2}+\|\operatorname{tg}(t)\|_{2}^{2}=\frac{1}{2} \sum_{n=0}^{\infty}\left[(2 n+1)\left(1+\theta_{n}^{2}\right)\left|\gamma_{n}\right|^{2}+\sqrt{n(n+1)}\left(1+\theta_{n-1} \theta_{n+1}\right)\left(\overline{\gamma_{n-1}} \gamma_{n+1}+\gamma_{n-1} \overline{\gamma_{n+1}}\right)\right] .
$$

Proof. We will use the recurrence relation (14), admitting that $\psi_{-1}=0$. So, if we consider that $\gamma_{-1}=0$, we obtain

$$
\sqrt{2}\left\langle t f(t), \psi_{n}(t)\right\rangle_{2}=\sqrt{n+1} \gamma_{n+1}+\sqrt{n} \gamma_{n-1}, \quad n \in \mathbb{N} .
$$

Admitting that $\theta_{-1}=1$ and using (16)-(17), we have

$$
\begin{aligned}
\sqrt{2}\left\langle\operatorname{tg}(t), \psi_{n}(t)\right\rangle_{2} & =\left\langle g(t), \sqrt{2} t \psi_{n}(t)\right\rangle_{2}=\left\langle g(t), \sqrt{n+1} \psi_{n+1}(t)+\sqrt{n} \psi_{n-1}(t)\right\rangle_{2} \\
& =\sqrt{n+1}\left\langle g(t), \psi_{n+1}(t)\right\rangle_{2}+\sqrt{n}\left\langle g(t), \psi_{n-1}(t)\right\rangle_{2}=\sqrt{n+1} \theta_{n+1} \gamma_{n+1}+\sqrt{n} \theta_{n-1} \gamma_{n-1} .
\end{aligned}
$$


Hence,

$$
\begin{aligned}
\|t f(t)\|_{2}^{2} & =\langle t f(t), t f(t)\rangle_{2}=\frac{1}{2} \sum_{n=0}^{\infty}\left|\sqrt{n+1} \gamma_{n+1}+\sqrt{n} \gamma_{n-1}\right|^{2} \\
& =\frac{1}{2} \sum_{n=0}^{\infty}\left[(n+1)\left|\gamma_{n+1}\right|^{2}+n\left|\gamma_{n-1}\right|^{2}+\sqrt{n(n+1)}\left(\overline{\gamma_{n-1}} \gamma_{n+1}+\gamma_{n-1} \overline{\gamma_{n+1}}\right)\right] \\
& =\frac{1}{2} \sum_{n=0}^{\infty}(n+1)\left|\gamma_{n+1}\right|^{2}+\frac{1}{2} \sum_{n=0}^{\infty} n\left|\gamma_{n-1}\right|^{2}+\frac{1}{2} \sum_{n=0}^{\infty} \sqrt{n(n+1)}\left(\overline{\gamma_{n-1}} \gamma_{n+1}+\gamma_{n-1} \overline{\gamma_{n+1}}\right) .
\end{aligned}
$$

Let $k:=n+1$ and $i:=n-1$. So, we obtain

$$
\begin{aligned}
\|t f(t)\|_{2}^{2} & =\frac{1}{2} \sum_{k=1}^{\infty} k\left|\gamma_{k}\right|^{2}+\frac{1}{2} \sum_{i=0}^{\infty}(i+1)\left|\gamma_{i}\right|^{2}+\frac{1}{2} \sum_{n=0}^{\infty} \sqrt{n(n+1)}\left(\overline{\gamma_{n-1}} \gamma_{n+1}+\gamma_{n-1} \overline{\gamma_{n+1}}\right) \\
& =\frac{1}{2} \sum_{n=0}^{\infty} n\left|\gamma_{n}\right|^{2}+\frac{1}{2} \sum_{n=0}^{\infty}(n+1)\left|\gamma_{n}\right|^{2}+\frac{1}{2} \sum_{n=0}^{\infty} \sqrt{n(n+1)}\left(\overline{\gamma_{n-1}} \gamma_{n+1}+\gamma_{n-1} \overline{\gamma_{n+1}}\right) \\
& =\frac{1}{2} \sum_{n=0}^{\infty}\left[(2 n+1)\left|\gamma_{n}\right|^{2}+\sqrt{n(n+1)}\left(\overline{\gamma_{n-1}} \gamma_{n+1}+\gamma_{n-1} \overline{\gamma_{n+1}}\right)\right] .
\end{aligned}
$$

Besides this, we have

$$
\begin{aligned}
\|\operatorname{tg}(t)\|_{2}^{2} & =\langle\operatorname{tg}(t), \operatorname{tg}(t)\rangle_{2}=\frac{1}{2} \sum_{n=0}^{\infty}\left[(n+1) \theta_{n+1}^{2}\left|\gamma_{n+1}\right|^{2}+n \theta_{n-1}^{2}\left|\gamma_{n-1}\right|^{2}+\sqrt{n(n+1)} \theta_{n-1} \theta_{n+1}\left(\overline{\gamma_{n-1}} \gamma_{n+1}+\gamma_{n-1} \overline{\gamma_{n+1}}\right)\right] \\
& =\frac{1}{2} \sum_{n=0}^{\infty}\left[(2 n+1) \theta_{n}^{2}\left|\gamma_{n}\right|^{2}+\sqrt{n(n+1)} \theta_{n-1} \theta_{n+1}\left(\overline{\gamma_{n-1}} \gamma_{n+1}+\gamma_{n-1} \overline{\gamma_{n+1}}\right)\right]
\end{aligned}
$$

Adding (20) and (21), we obtain (18).

Lemma 16 The inequality $4\|t f(t)\|_{2}^{2}+\|\operatorname{tg}(t)\|_{2}^{2} \geq 4\|f\|_{2}^{2}$ holds true for $f \in L^{2}(\mathbb{R})$ and $g=T f$.

Proof. From the previous lemma, we have that

$$
\begin{aligned}
\|t f(t)\|_{2}^{2}+\|t g(t)\|_{2}^{2}= & \frac{1}{2} \sum_{n=0}^{\infty}\left[(2 n+1)\left(1+\theta_{n}^{2}\right)\left|\gamma_{n}\right|^{2}+\sqrt{n(n+1)}\left(1+\theta_{n-1} \theta_{n+1}\right)\left(\overline{\gamma_{n-1}} \gamma_{n+1}+\gamma_{n-1} \overline{\gamma_{n+1}}\right)\right] \\
= & \frac{1}{2} \sum_{n=0}^{\infty}\left[(2 n+1)\left(1+\theta_{n}^{2}\right)\left|\gamma_{n}\right|^{2}+\left(1+\theta_{n-1} \theta_{n+1}\right)\left|\sqrt{n+1} \gamma_{n+1}+\sqrt{n} \gamma_{n-1}\right|^{2}\right] \\
& -\frac{1}{2} \sum_{n=0}^{\infty}\left(1+\theta_{n-1} \theta_{n+1}\right)\left[(n+1)\left|\gamma_{n+1}\right|^{2}+n\left|\gamma_{n-1}\right|^{2}\right] .
\end{aligned}
$$

Doing $n:=n+1$ and $n:=n-1$, we obtain

$$
\begin{aligned}
\|t f(t)\|_{2}^{2}+\|t g(t)\|_{2}^{2}= & \frac{1}{2} \sum_{n=0}^{\infty}\left[(2 n+1)\left(1+\theta_{n}^{2}\right)\left|\gamma_{n}\right|^{2}+\left(1+\theta_{n-1} \theta_{n+1}\right)\left|\sqrt{n+1} \gamma_{n+1}+\sqrt{n} \gamma_{n-1}\right|^{2}\right] \\
& -\frac{1}{2} \sum_{n=0}^{\infty}(2 n+1)\left(1+\theta_{n} \theta_{n+2}\right)\left|\gamma_{n}\right|^{2} \\
= & \sum_{n=0}^{\infty}\left[(2 n+1) \theta_{n}^{2}\left|\gamma_{n}\right|^{2}+\frac{1}{2} \sum_{n=0}^{\infty}\left(1-\theta_{n-1}^{2}\right)\left|\sqrt{n+1} \gamma_{n+1}+\sqrt{n} \gamma_{n-1}\right|^{2}\right] \\
= & 4\left|\gamma_{0}\right|^{2}+3\left|\gamma_{1}\right|^{2}+\sum_{n=2}^{\infty}(2 n+1) \theta_{n}^{2}\left|\gamma_{n}\right|^{2}-\frac{1}{2} \sum_{n=1}^{\infty}\left(1-\theta_{n-1}^{2}\right)\left|\sqrt{n+1} \gamma_{n+1}+\sqrt{n} \gamma_{n-1}\right|^{2} .
\end{aligned}
$$


As $1-\theta_{n}^{2} \geq-3$ and $3\left|\gamma_{1}\right|^{2} \geq 4\left|\gamma_{1}\right|^{2}-\frac{3}{2}\left|\gamma_{1}\right|^{2}$, we have

$$
\begin{aligned}
\|t f(t)\|_{2}^{2}+\|t g(t)\|_{2}^{2} & \geq 4\left|\gamma_{0}\right|^{2}+4\left|\gamma_{1}\right|^{2}+4 \sum_{n=2}^{\infty}\left|\gamma_{n}\right|^{2}-\frac{3}{2}\left|\gamma_{1}\right|^{2}-\frac{3}{2} \sum_{n=1}^{\infty}\left|\sqrt{n+1} \gamma_{n+1}+\sqrt{n} \gamma_{n-1}\right|^{2} \\
& =4 \sum_{n=0}^{\infty}\left|\gamma_{n}\right|^{2}-\frac{3}{2} \sum_{n=0}^{\infty}\left|\sqrt{n+1} \gamma_{n+1}+\sqrt{n} \gamma_{n-1}\right|^{2}=4 \int_{\mathbb{R}}|f(t)|^{2} d t-3 \int_{\mathbb{R}} t^{2}|f(t)|^{2} d t .
\end{aligned}
$$

In this way, we can say that $4\|t f(t)\|_{2}^{2}+\|\operatorname{tg}(t)\|_{2}^{2} \geq 4\|f\|_{2}^{2}$.

After the previous preparatory results, we are now in condition to present the proof of Theorem 14.

Proof. For some $p>0$, we will consider the following functions $f_{1}(t):=p^{-\frac{1}{2}} f\left(\frac{t}{p}\right)$ and $g_{1}(t):=p^{\frac{1}{2}} g(t p)$. We can prove that $g_{1}=T f_{1}$. Indeed,

$$
\begin{aligned}
\left(T f_{1}\right)(t) & =\frac{1}{\sqrt{2 \pi}} \int_{\mathbb{R}}[2 \cos (t y)+i \sin (t y)] f_{1}(y) d y=\frac{1}{2} \frac{1}{\sqrt{2 \pi}} \int_{\mathbb{R}} e^{-i t y} p^{-\frac{1}{2}} f\left(\frac{y}{p}\right) d y+\frac{3}{2} \frac{1}{\sqrt{2 \pi}} \int_{\mathbb{R}} e^{i t y} p^{-\frac{1}{2}} f\left(\frac{y}{p}\right) d y \\
& =p^{\frac{1}{2}}\left[\frac{1}{2} \frac{1}{\sqrt{2 \pi}} \int_{\mathbb{R}} e^{-i t p y} f(y) d y+\frac{3}{2} \frac{1}{\sqrt{2 \pi}} \int_{\mathbb{R}} e^{i t p y} f(y) d y\right]=p^{\frac{1}{2}}(T f)(t p)=p^{\frac{1}{2}} g(t p)=g_{1}(t) .
\end{aligned}
$$

Applying Lemma 16 for $f_{1}$ and $g_{1}$, we obtain $4\left\|t f_{1}(t)\right\|_{2}^{2}+\left\|t g_{1}(t)\right\|_{2}^{2} \geq 4\left\|f_{1}\right\|_{2}^{2}$, which is equivalent to

$$
4 p^{2}\|t f(t)\|_{2}^{2}+p^{-2}\|\operatorname{tg}(t)\|_{2}^{2} \geq 4\left\|f_{1}\right\|_{2}^{2}=4\|f\|_{2}^{2} .
$$

Taking the minimum on the left-hand side, with respect to the variable $p \in(0,+\infty)$, we have

$$
\min _{p \in(0,+\infty)}\left\{4 p^{2}\|t f(t)\|_{2}^{2}+p^{-2}\|t g(t)\|_{2}^{2}\right\}=4\|t f(t)\|_{2} \cdot\|t g(t)\|_{2} .
$$

So, we obtain that $4\|t f(t)\|_{2} \cdot\|t g(t)\|_{2} \geq 4\|f\|_{2}^{2}$, which is equivalent to (15).

Theorem 17 A generalization of Theorem 14 holds true for any $f \in L^{2}\left(\mathbb{R}^{d}\right)$. Namely,

$$
\left(\int_{\mathbb{R}^{d}}|x-a|^{2}|f(x)|^{2} d x\right)^{1 / 2}\left(\int_{\mathbb{R}^{d}}|\xi-b|^{2}|T f(\xi)|^{2} d \xi\right)^{1 / 2} \geq d \int_{\mathbb{R}^{d}}|f(x)|^{2} d x
$$

where $a, b \in \mathbb{R}^{d}$ are arbitrary vectors.

Proof. Notice that by a simple changing of variables (or, equivalently, a shift of the axes $O x_{k}, O \xi, k=1, \ldots, d$ ), we can assume that $a=b=0$. Put

$$
g\left(x_{2}, \ldots, x_{d}\right):=\left(\int_{\mathbb{R}} x_{1}^{2}|f(x)|^{2} d x_{1}\right)^{1 / 2}, \quad G\left(x_{2}, \ldots, x_{d}\right):=\left(\int_{\mathbb{R}} x_{1}^{2}|T f(x)|^{2} d x_{1}\right)^{1 / 2},
$$

in which $g$ and $G$ are positive functions of variables $x_{2}, \ldots, x_{d}$. On the other hand, for a convenient use of some integral inequalities, we will unify the symbols $x$ and $\xi$ in the inequality (26). Having in mind that in the integrals of (27), $x_{2}, \ldots, x_{d}$ are considered as parameters, and using the one-dimensional version of the uncertainty inequality already obtained in Theorem 14, we have

$$
\left(\int_{\mathbb{R}} x_{1}^{2}|f(x)|^{2} d x_{1}\right)^{1 / 2}\left(\int_{\mathbb{R}} x_{1}^{2}|T f(x)|^{2} d x_{1}\right)^{1 / 2} \geq \int_{\mathbb{R}}|f(x)|^{2} d x_{1} .
$$


By considering the consequent iterated integrals, the Cauchy-Schwartz inequality (C-S inequality) and (28), we have

$$
\begin{aligned}
& \left(\int_{\mathbb{R}^{d}} x_{1}^{2}|f(x)|^{2} d x_{1} \cdots d x_{d}\right)\left(\int_{\mathbb{R}^{d}} x_{1}^{2}|(T f)(x)|^{2} d x_{1} \cdots d x_{d}\right) \\
& =\underbrace{\left(\int_{\mathbb{R}^{d-1}}\left|g\left(x_{2}, \ldots, x_{d}\right)\right|^{2} d x_{2} \cdots d x_{d}\right)\left(\int_{\mathbb{R}^{d-1}}\left|G\left(x_{2}, \ldots, x_{d}\right)\right|^{2} d x_{2} \cdots d x_{d}\right)}_{\text {using the C-S inequality }} \geq\left(\int_{\mathbb{R}^{d-1}} g\left(x_{2}, \ldots, x_{d}\right) G\left(x_{2}, \ldots x_{d}\right) d x_{2} \ldots d x_{d}\right)^{2} \\
& =\left(\int_{\mathbb{R}^{d-1}}[\underbrace{\left(\int_{\mathbb{R}} x_{1}^{2}|f(x)|^{2} d x_{1}\right)^{1 / 2}\left(\int_{\mathbb{R}} x_{1}^{2}|T f(x)|^{2} d x_{1}\right)^{1 / 2}}_{\text {using }(28)}] d x_{2} \cdots d x_{d}\right)^{2} \geq\left(\int_{\mathbb{R}^{d}}|f(x)|^{2} d x_{1} d x_{2} \cdots d x_{d}\right)^{2}=\|f\|_{2}^{4} .
\end{aligned}
$$

Similarly, we can prove that

$$
\left(\int_{\mathbb{R}^{d}} x_{k}^{2}|f(x)|^{2} d x\right)^{1 / 2}\left(\int_{\mathbb{R}^{d}} x_{k}^{2}|(T f)(x)|^{2} d x\right)^{1 / 2} \geq\|f\|_{2}^{2}, \quad \text { for every } k=1, \ldots, d .
$$

Taking both sides of (29) and summing over $k$, and using the Cauchy-Schwartz inequality of an inner product of vectors in $\mathbb{R}^{d}$, we obtain at once the $d$-dimensional form of the Heisenberg's inequality. Indeed, we have

$$
\begin{aligned}
d\|f\|_{2}^{2} & \leq \sum_{k=1}^{d} \underbrace{\left(\int_{\mathbb{R}^{d}} x_{k}^{2}|f(x)|^{2} d x\right)^{1 / 2}}_{\text {putting as } A_{k}} \underbrace{\left(\int_{\mathbb{R}^{d}} x_{k}^{2}|(T f)(x)|^{2} d x\right)^{1 / 2}}_{\text {putting as } B_{k}} \\
& \leq(\sum_{k=1}^{d} \underbrace{\left[\int_{\mathbb{R}^{d}} x_{k}^{2}|f(x)|^{2} d x\right]}_{A_{k}^{2}})^{1 / 2}\left(\sum_{k=1}^{d}[\underbrace{\left[\int_{\mathbb{R}^{d}} x_{k}^{2}|T f(x)|^{2} d x\right]}_{B_{k}^{2}})^{1 / 2}=\left(\int_{\mathbb{R}^{d}}|x|^{2}|f(x)|^{2} d x\right)^{1 / 2}\left(\int_{\mathbb{R}^{d}}|x|^{2}|T f(x)|^{2} d x\right)^{1 / 2},\right.
\end{aligned}
$$

as desired. The theorem is proved.

Another uncertainty principle of Heisenberg type for our operator $T$, also for the $\mathbb{R}^{d}$ case, is presented in the following theorem.

Theorem 18 If $\psi \in L^{2}\left(\mathbb{R}^{d}\right)$, then

$$
\|x \psi(x)\|_{2}\left[-\left\langle y(T \psi)(y), y\left(T^{-1} \psi\right)(-y)\right\rangle_{2}\right]^{\frac{1}{2}} \geq \frac{1}{2}\|\psi\|_{2}^{2} .
$$

Proof. We will start with the case $d=1$. Let $\psi, \psi^{\prime} \in \mathcal{S}$. Integrating by parts, we have

$$
\|\psi\|_{2}^{2}=\int_{\mathbb{R}}|\psi(x)|^{2} d x=-\int_{\mathbb{R}} x \frac{d}{d x}|\psi(x)|^{2} d x=-\left\langle x \psi^{\prime}(x), \psi(x)\right\rangle_{2}-\left\langle x \psi(x), \psi^{\prime}(x)\right\rangle_{2} .
$$

Using the fact that $\left|\left\langle x \psi^{\prime}(x), \psi(x)\right\rangle_{2}\right|=\left|\left\langle x \psi(x), \psi^{\prime}(x)\right\rangle_{2}\right|$ and the Cauchy-Schwartz inequality, we obtain that

$$
\|\psi\|_{2}^{2} \leq 2 \int_{\mathbb{R}}\left|x\|\psi(x)\| \psi^{\prime}(x)\right| d x \leq 2\|x \psi(x)\|_{2}\left\|\psi^{\prime}(x)\right\|_{2}
$$

By Theorem 8 , we can write $\psi=\left(T^{-1}(T \psi)\right)$. So,

$$
\psi^{\prime}(x)=\frac{1}{\sqrt{2 \pi}} \int_{\mathbb{R}}\left[-\frac{y}{2} \sin (x y)-i y \cos (x y)\right](T \psi)(y) d y=\frac{1}{\sqrt{2 \pi}} \int_{\mathbb{R}} y\left[-\frac{1}{2} \sin (x y)-i \cos (x y)\right](T \psi)(y) d y .
$$


Let $R(x):=\frac{1}{\sqrt{2 \pi}} \int_{\mathbb{R}}\left[-\frac{1}{2} \sin (x y)-i \cos (x y)\right] f(y) d y, f \in L^{2}(\mathbb{R})$. Thus, $\psi^{\prime}(x)=(R(y(T \psi)(y)))(x)$. We can now rewrite $R$ in the form $R=-\frac{3 i}{4} F-\frac{i}{4} F^{-1}$ so that we immediately obtain $\langle R f, R g\rangle_{2}=\frac{3}{8}\langle f, g\rangle_{2}+\frac{5}{8}\langle f, W g\rangle_{2}$. Hence, we have

$$
\begin{aligned}
\left\|\psi^{\prime}\right\|_{2}^{2}= & \|R(y(T \psi)(y))\|_{2}^{2}=\frac{3}{8}\langle y(T \psi)(y), y(T \psi)(y)\rangle_{2}-\frac{5}{8}\langle y(T \psi)(y), y(T \psi)(-y)\rangle_{2} \\
= & \frac{1}{8}\langle y(T \psi)(y), 3 y(T \psi)(y)-5 y(T \psi)(-y)\rangle_{2}=\frac{1}{8} \frac{1}{2 \pi} \int_{\mathbb{R}} \int_{\mathbb{R}} y(2 \cos (y t)+i \sin (y t)) \psi(t) \\
& {\left[3 \int_{\mathbb{R}}(y(2 \cos (y v)-i \sin (y v))) \overline{\psi(v)} d v-5 \int_{\mathbb{R}}(y(2 \cos (y v)+i \sin (y v))) \overline{\psi(v)} d v\right] d t d y } \\
= & -\frac{1}{2 \pi} \int_{\mathbb{R}} \int_{\mathbb{R}} \int_{\mathbb{R}} y^{2}(\cos (y t)+i \sin (y t))\left(\frac{1}{2} \cos (y v)+i \sin (y v)\right) \psi(v) \overline{\psi(v)} d v d t d y \\
= & -\left\langle y(T \psi)(y), y\left(T^{-1} \psi\right)(-y)\right\rangle_{2} .
\end{aligned}
$$

Then, $\|\psi\|_{2}^{2} \leq 2\|x \psi(x)\|_{2}\left\|\psi^{\prime}\right\|_{2}=2\|x \psi(x)\|_{2}\left[-\left\langle y(T \psi)(y), y\left(T^{-1} \psi\right)(-y)\right\rangle_{2}\right]^{\frac{1}{2}}$, which is equivalent to (30), in the case $d=1$.

For $d>1$, (30) follows from the case $d=1$, by using the same method as in the proof of Theorem 17, and so we avoid to present the corresponding detailed full proof in here.

\section{ACKNOWLEDGMENTS}

This work was supported in part by FCT-Portuguese Foundation for Science and Technology through the Center for Research and Development in Mathematics and Applications (CIDMA) of Universidade de Aveiro, within project UID/MAT/04106/2013. R. C. Guerra also acknowledges the direct support of the Portuguese Foundation for Science and Technology (FCT) through the scholarship PD/BD/114187/2016.

\section{REFERENCES}

[1] L. Debnath and D. Bhatta, Integral Transforms and their Applications, 2nd ed. (Chapman \& Hall/CRC, Boca Raton, 2007).

[2] C. Fefferman, "The uncertainty principle”, Bull. Amer. Math. Soc. (N.S.) 9, 129-206 (1983).

[3] G.B. Folland and A. Sitaram, "The uncertainty principle: a mathematical survey", J. Fourier Anal. Appl. 3 (3), 207-238 (1997).

[4] D. Gabor, "Theory of communication”, J. Inst. Elec. Engr. 93, 429-457 (1946).

[5] W. Heisenberg, "Über den anschaulichen Inhalt der quantentheoretischen Kinematic und Mechanik", Zeit. Physik 43, 172-198 (1927).

[6] M. F. E. de Jeu, "An uncertainty principle for integral operators", J. Functional Analysis 22, 247-253 (1994).

[7] E. H. Kennard, "Zur Quantenmechanik einfacher Bewegungstypen", Zeit. Physik 44, 326-352 (1927).

[8] H. P. Robertson, "The uncertainty principle", Phys. Rev. 34, 573-574 (1929); reprinted in Wheeler and Zurek, 127-128 (1983).

[9] W. Rudin, Functional Analysis (McGraw-Hill, New York, 1991).

[10] E. Schrödinger, "Zum Heisenbergschen Unschärfeprinzip”, Sitzungsber. Preuß. Akad. Wiss., Phys.-Math. Kl., 296-303 (1930); Proceedings Royal Irish Acad. (in English) 39 (A), 73-81 (1930).

[11] D. Slepian, "Some comments on Fourier analysis, uncertainty and modeling", SIAM Rev. 25, 379-393 (1983).

[12] K. T. Smith, "The uncertainty principle on groups", SIAM J. Appl. Math. 50, 876-882 (1990).

[13] N. Sochen, P. Maass, C. Sagiv and H. G. Stark, "Do uncertainty minimizers attain minimal uncertainty?", J. Fourier Anal. Appl. 16, 448-469 (2010).

[14] E. M. Stein and R. Shakarchi, Fourier Analysis. An Introduction, Princeton Lectures in Analysis, 1 (Princeton University Press, Princeton and Oxford, 2003).

[15] S. Thangavelu, Harmonic Analysis on the Heisenberg Group (Springer, New York, 1998).

[16] E. C. Titchmarsh, Introduction to the Theory of Fourier Integrals (Chelsea Publ. Co., New York, 1986).

[17] N. M. Tuan and P. D. Tuan, "Operator properties and Heisenberg uncertainty principles for a un-unitary integral operator", Integral Transforms Spec. Funct. 23 (1), 1-12 (2012). 